Condensed Matter Physics, 1998, Vol. 1, No 1(13), p. 11-22

\title{
Symmetries in the physics of strongly correlated electronic systems
}

\author{
F.Mancini, A.Avella \\ Università degli Studi di Salerno - Unità INFM di Salerno \\ Dipartimento di Scienze Fisiche "E.R. Caianiello" \\ 84081 Baronissi, Salerno, Italy
}

Received June 11, 1998

Strongly correlated electron systems require the development of new theoretical schemes in order to describe their unusual and unexpected properties. The usual perturbation schemes are inadequate and new concepts must be introduced. In our scheme of calculations, the Composite Operator Method is possible to recover through a self-consistent calculation, a series of fundamental symmetries by choosing a suitable Hilbert space.

Key words: strongly correlated electron systems, Hubbard model, symmetries, composite operator method

PACS: $71.10 .-w, 71.10 . F d, 71.27 .+a$

The discovery of new materials with a large variety of unusual and unexpected properties [1] has opened a new era in the physics of Condensed Matter; new theoretical schemes must be developed [2]. The most important characteristic of these new systems is a strong correlation among the electrons that makes classical schemes based on the band picture inapplicable. It is necessary to pass from singleelectron physics to many-electron physics where the dominant part will be the correlations among the electrons. Usual perturbation schemes are inadequate and new concepts must be introduced.

Let us consider a certain Hamiltonian

$$
H=H\left[\varphi_{1}(x), \ldots, \varphi_{n}(x)\right]
$$

where set $\left\{\varphi_{i}\right\}$ denotes band-electron fields. Due to strong correlation effects the properties of the original electrons $\left\{\varphi_{i}\right\}$ are drastically changed; new excitation modes will appear and determine most of the observed properties of the system. It is natural to identify a new set of elementary excitations $\left\{\psi_{i}\right\}$ as the basis for perturbative schemes. These excitations, constructed from the original electron set (in this sense we call them composite fields), are created by interactions among the electrons; therefore, their properties will be determined by the dynamics, the 
symmetries of the model, the boundary conditions and must be computed in a selfconsistent way [3]. This aspect enriches the theory and will allow us to realize the dynamics in the proper Hilbert space where the physical symmetries are conserved. On the other hand, we know from experiments that highly correlated systems exhibit an incredible variety of behaviours. It would be very hard to describe such a complexity using the original fields, unless the exact solution of the model is available. The presence of new excitations and composite fields introduces into the theory the possibility to accommodate the multifariousness of experimental properties.

A theory built on the basis of new excitation modes is, by construction, a selfconsistent theory, and the procedure must be fixed. In particular, we must consider the following list of questions:

1. The identification of the fundamental set;

2. The statistics and the properties of the new fields;

3. The symmetry and the dynamics in terms of the new fields;

4. The representation where the new fields are realized.

We will now try to formulate a scheme of calculations in which the answers to the above questions can be found. Then, by considering a particular model, we will present a practical realization of the theoretical scheme.

The new fields $\left\{\psi_{i}\right\}$ are generated by interactions among the bare fields; then, it is naturally to choose a new set which naturally appears through the equations of motion. The evolution of the original fields is described by the Heisenberg equation

$$
i \frac{\partial}{\partial t} \varphi_{i}(x)=\left[\varphi_{i}(x), H\right]=J_{i}[\varphi(x)] .
$$

Such an equation generates new fields $\left\{J_{i}[\varphi(x)]\right\}$ constructed as combinations of the bare fields. By starting from these fields and by considering new Heisenberg equations

$$
i \frac{\partial}{\partial t} \psi_{i}(x)=\left[\psi_{i}(x), H\right]
$$

we generate an infinite hierarchy of composite fields. It is naturally impossible to solve the infinite system of equations and some truncation procedure must be adopted. Let us consider an $n$-component field

$$
\psi=\left(\begin{array}{c}
\psi_{1} \\
\vdots \\
\psi_{n}
\end{array}\right)
$$

and let us choose the first $n-1$ fields such as

$$
i \frac{\partial}{\partial t} \psi_{i}(x)=\left[\psi_{i}(x), H\right]=\sum_{j=1}^{i+1} \gamma_{i j}(-i \nabla) \psi_{j}(x) \quad 1 \leqslant j \leqslant n-1
$$


The $n$ th-field $\psi_{n}(x)$ is determined by the field equation $\psi_{n-1}(x)$. The matrix $\gamma(-i \nabla)$ is completely determined by the dynamics. Then, we linearize the Heisenberg equation by writing

$$
i \frac{\partial}{\partial t} \psi(x)=\varepsilon(-i \nabla) \psi(x)
$$

where the eigenvalue or energy matrix $\varepsilon$ is self-consistently calculated by means of the equation

$$
\varepsilon\left(-i \nabla_{x}\right)\left\langle\left\{\psi(\boldsymbol{x}, t), \psi^{\dagger}(\boldsymbol{y}, t)\right\}\right\rangle=\left\langle\left\{[\psi(\boldsymbol{x}, t), H], \psi^{\dagger}(\boldsymbol{y}, t)\right\}\right\rangle .
$$

Symbol $\langle\cdots\rangle$ denotes the thermal average. Derivative operators $\lambda(-i \nabla)$ are defined through the relation

$$
\lambda(-i \nabla) f(\boldsymbol{x})=\int d^{d} y \lambda(\boldsymbol{x}, \boldsymbol{y}) f(\boldsymbol{y}) .
$$

The rank of the energy matrix is equal to $n$, the number of components of vector $\psi(x)$. When there is translational invariance, we can invert equation (7) and it is easy to see that

$$
\begin{array}{r}
\varepsilon_{i j}=\left\langle\left\{\left[\psi_{i}(x), H\right], \psi_{l}^{\dagger}(y)\right\}\right\rangle\left\langle\left\{\psi_{l}(x), \psi_{j}^{\dagger}(y)\right\}\right\rangle^{-1}=\gamma_{i j} \\
1 \leqslant i \leqslant n-1, \quad 1 \leqslant j \leqslant n .
\end{array}
$$

This approximation corresponds to the $n$-pole expansion of the Green function where finite life-time contributions are neglected. It has been proved [4] that in this approximation the choice (5) of the composite operators leads to the conservation of spectral moments. In particular, the first $2(n-i+1)$ spectral moments for the field $\psi_{i}[1 \leqslant i \leqslant n-1]$ are conserved. This is an important property when we recall that the spectral moments are related to the spectral density function of singleparticle propagators. Also, as shown in Ref. [4], choice (5) leads to an equivalence between the $n$-pole approximation and the spectral density approach [5], although very different results are obtained when different procedures for self-consistency are used [6].

In general, the composite fields will not satisfy canonical anticommutation relations and their algebra must be calculated starting from the canonical algebra of the electron fields. Owing to this fact, the Wick theorem and the standard perturbation schemes cannot be applied. Examples of the new algebra will be presented further in this article.

The properties of the new fields are fixed by a series of parameters which must be self-consistently calculated. These parameters are expressed as expectation values of composite fields. When the composite fields belong to the set they can be expressed in terms of a single-particle Green function and calculated by a series of coupled self-consistent equations.

However, it may happen that some of the parameters are expressed as expectation values of higher-order composite fields that do not belong to the basic set. In 
this case, owing to the approximation considered, the parameters are not strictly bound by the dynamics and there is a freedom in the procedure to fix them. At this level the powerfulness of the scheme manifests itself: one can use this freedom to choose the right representation. In the construction of a physical theory we must distinguish two levels. On the one hand, we have a microscopic level where we are concerned with particles. The basic ingredients are the Heisenberg fields which together with the canonical commutation relations describe the dynamics. The physical laws (the equations of motion, the conservation laws, the symmetry principles) are expressed as relations among the operators. On the other hand, we have the macroscopic world where we are concerned with the average values of operators. At the level of observation the physical laws manifest themselves as relations among matrix elements, and a suitable choice of the Hilbert space must be made. When some approximation is introduced, the states are not the exact eigenstates of the Hamiltonian; the expectation values are also not the exact ones. As a consequence, the relations among the operators are generally not conserved when the expectation values are calculated. A striking example of this is the violation of the Pauli principle. A convenient way to take care of it is to operate in the representation of the second quantization where the Pauli principle manifests itself through the algebra. It is known [7] that in most of the approximation schemes this symmetry is violated when matrix elements are considered. Other examples of symmetries will be considered later.

The point of view adopted in this approach is that we can use the freedom in the procedure of fixing the self-consistent parameters in order to recover the symmetries violated by the approximation. In general, the model exhibits many different symmetries and there will be a relation between the number of composite fields and the number of symmetries that can be recovered. On the physical ground one must choose which symmetries are the most important to be satisfied. In a physics dominated by strong electron correlations the Pauli principle plays a crucial role, and it is extremely relevant that the related symmetries be treated in a correct way. Therefore, in our scheme the attention is firstly paid to the Pauli principle; once this is done, the attention is devoted to other symmetries.

As an illustration of the scheme we shall now consider the Hubbard model [8]. In the standard notation this model is described by the following Hamiltonian:

$$
H=\sum_{i j}\left(t_{i j}-\mu \delta_{i j}\right) c^{\dagger}(i) c(i)+U \sum_{i} n_{\uparrow}(i) n_{\downarrow}(i)
$$

$c(i), c^{\dagger}(i)$ are annihilation and creation operators for electrons at site $i$ in the spinor notation

$$
c(i)=\left(\begin{array}{c}
c_{\uparrow}(i) \\
c_{\downarrow}(i)
\end{array}\right), \quad c^{\dagger}(i)=\left(c_{\uparrow}^{\dagger}(i), c_{\downarrow}^{\dagger}(i)\right),
$$

$n_{\sigma}=c_{\sigma}^{\dagger}(i) c_{\sigma}(i)$ is the number operator of electrons with spin $\sigma=(\uparrow, \downarrow)$ at the $i^{t h}$ site, $\mu$ is the chemical potential and is introduced in order to control the band filling $n$. For a two-dimensional squared lattice and by restricting the analysis to 
first nearest neighbours, the hopping matrix $t_{i j}$ has the form:

$$
\begin{aligned}
t_{i j} & =-4 t \alpha_{i j}=-4 t \frac{1}{N} \sum_{k} e^{i \boldsymbol{k} \cdot\left(\boldsymbol{R}_{i}-\boldsymbol{R}_{j}\right)} \alpha(\boldsymbol{k}), \\
\alpha(\boldsymbol{k}) & =\frac{1}{2}\left[\cos \left(k_{x} a\right)+\cos \left(k_{y} a\right)\right]
\end{aligned}
$$

$a$ being the lattice constant. In addition to the band term, the model contains an interaction term which approximates the interaction among the electrons. In the simplest form of the Hubbard model, the interaction is between electrons of the opposite spin on the same lattice site; the strength of the interaction is described by parameter $U$.

The electron field $c(i)$ satisfies the Heisenberg equation

$$
i \frac{\partial}{\partial t} c(i)=-\mu c(i)-4 t c^{\alpha}(i)+U \eta(i)
$$

where

$$
c^{\alpha}(i)=\sum_{j} \alpha_{i j} c(j)
$$

is an electron field on the nearest neighbour sites. We see that the dynamics has generated the composite field

$$
\eta(i)=c(i) n(i)
$$

The Heisenberg equation for this field will generate a new higher-order composite field. The process does not stop and an infinite number of composite fields will be generated. By following the procedure mentioned above, we close the hierarchy by considering $\mathrm{n}$ fields and construct a vector composite field as described by Eqs. (4) and (5). For the specific case, we consider the three-component field

$$
\psi(i)=\left(\begin{array}{l}
\psi_{1}(i) \\
\psi_{2}(i) \\
\psi_{3}(i)
\end{array}\right)
$$

where

$$
\begin{aligned}
& \psi_{1}(i)=\xi(i)=c(i)[1-n(i)] \\
& \psi_{2}(i)=\eta(i)=c(i) n(i) \\
& \psi_{3}(i)=\pi(i)=\frac{1}{2} \sigma^{\mu} n_{\mu}(i) c^{\alpha}(i)+c(i) c^{\dagger \alpha}(i) c(i),
\end{aligned}
$$

$n_{\mu}(i)=c^{\dagger}(i) \sigma_{\mu} c(i)$ is the charge $(\mu=0)$ and spin $(\mu=1,2,3)$ density operator for $c$-electrons. We are using the following notation $\sigma_{\mu}=(1, \boldsymbol{\sigma}), \sigma^{\mu}=(-1, \boldsymbol{\sigma})$, $\boldsymbol{\sigma}$ being the Pauli matrices. The composite fields (17) do not satisfy a canonical 
algebra. For example, for the first two fields

$$
\begin{aligned}
\left\{\xi(i), \xi^{\dagger}(j)\right\} & =\delta_{i j}\left[1-\frac{1}{2} \sigma^{\mu} n_{\mu}(i)\right], \\
\left\{\eta(i), \eta^{\dagger}(j)\right\} & =-\delta_{i j} \frac{1}{2} \sigma^{\mu} n_{\mu}(i), \\
\left\{\xi(i), \eta^{\dagger}(j)\right\} & =\{\xi(i), \xi(j)\}=\{\eta(i), \eta(j)\}=0 .
\end{aligned}
$$

This field satisfies the Heisenberg equation

$$
i \frac{\partial}{\partial t} \psi(i)=J(i)=\left(\begin{array}{c}
-\mu \xi(i)-4 t c^{\alpha}(i)-4 t \pi(i) \\
(-\mu+U) \eta(i)+4 t \pi(i) \\
-\mu \pi(i)+4 t \kappa(i)-4 t \theta(i)+U \rho(i)
\end{array}\right)
$$

where

$$
\begin{aligned}
\kappa(i) & =\frac{1}{2} \sigma^{\mu} c(i) c^{\dagger \alpha}(i) \sigma_{\mu} c^{\alpha}(i)-\frac{1}{2} \sigma^{\mu} c^{\alpha}(i) c^{\dagger}(i) \sigma_{\mu} c^{\alpha}(i), \\
\theta(i) & =\frac{1}{2} \sigma^{\mu} n_{\mu}(i) c^{\alpha^{2}}(i)+c^{\alpha}(i) c^{\dagger \alpha}(i) c(i)-c(i) c^{\dagger \alpha^{2}}(i) c(i)+c(i) c^{\dagger \alpha}(i) c^{\alpha}(i), \\
\rho(i) & =\frac{1}{2} \sigma^{\mu} n_{\mu}(i) \eta^{\alpha}(i)+c(i) \xi^{\dagger \alpha}(i) c(i) .
\end{aligned}
$$

According to the method presented above, the equation of motion (20) is linearized as

$$
i \frac{\partial}{\partial t} \psi(i)=\sum_{j} \varepsilon(i, j) \psi(j)
$$

where the energy matrix $\varepsilon(i, j)$ is the $3 \times 3$ matrix given by

$$
\varepsilon(i, j)=\left\langle\left\{J(i), \psi^{\dagger}(j)\right\}\right\rangle_{\text {E.T. }}\left\langle\left\{\psi(i), \psi^{\dagger}(j)\right\}\right\rangle_{\text {E.T. }}^{-1} .
$$

The subscript E.T. indicates that the anticommutators are evaluated at equal time.

The physical properties can be described in terms of the thermal retarded Green function

$$
S(i, j)=\left\langle R\left[\psi(i) \psi^{\dagger}(i)\right]\right\rangle=\frac{i a^{2}}{(2 \pi)^{3}} \int_{\Omega_{\mathrm{B}}} \mathrm{d}^{2} k \mathrm{~d} \omega e^{i \boldsymbol{k} \cdot\left(\boldsymbol{R}_{i}-\boldsymbol{R}_{j}\right)-i \omega\left(t_{i}-t_{j}\right)} S(\boldsymbol{k}, \omega),
$$

where $R$ is the usual retarded operator and symbol $\langle\cdots\rangle$ denotes the thermal average. By means of the linearized Heisenberg equation (22) the Fourier transform is given by

$$
S(\boldsymbol{k}, \omega)=\sum_{n=1}^{3} \frac{\sigma^{n}(\boldsymbol{k})}{\omega-E_{n}(\boldsymbol{k})+i \eta}
$$

where energy spectra $E_{i}(\boldsymbol{k})$ are the characteristic values of the matrix $\varepsilon(\boldsymbol{k})$, determined by the equation

$$
\sum_{m=1}^{3} a_{m}(\boldsymbol{k}) E_{n}^{m}(\boldsymbol{k})=0
$$


The characteristic coefficients $a_{i}(\boldsymbol{k})$ are defined by the following relation:

$$
a_{n-k}(\boldsymbol{k})=(-)^{k} \operatorname{Tr}_{k}[\varepsilon(\boldsymbol{k})] \quad 0 \leqslant k \leqslant 3
$$

where $\operatorname{Tr}_{k}$ is the trace of the $k^{\text {th }}$ order defined as a sum of determinants of all $\left(\begin{array}{l}3 \\ k\end{array}\right)$ matrices of order $k \times k$ which can be formed by intersecting any $k$ rows of $\varepsilon$ with the same $k$ columns. We note that $\operatorname{Tr}_{3}[\varepsilon]=\operatorname{Det}[\varepsilon]$ and the convention $\operatorname{Tr}_{0}[\varepsilon]=1$ is used. The spectral functions are given by

$$
\sigma^{n}(\boldsymbol{k})=\frac{1}{b_{n}(\boldsymbol{k})} \sum_{m=0}^{2} E_{n}^{m}(\boldsymbol{k}) \lambda^{m}(\boldsymbol{k})
$$

where the $\lambda^{n}(\boldsymbol{k})$ are $3 \times 3$ matrices:

$$
\lambda^{n}(\boldsymbol{k})=\sum_{m=n+1}^{3} a_{m}(\boldsymbol{k}) \varepsilon^{m-n-1}(\boldsymbol{k}) I(\boldsymbol{k}) \quad 0 \leqslant n \leqslant 2
$$

and we put

$$
b_{n}(\boldsymbol{k})=\prod_{m=1, m \neq n}^{3}\left[E_{n}(\boldsymbol{k})-E_{m}(\boldsymbol{k})\right]
$$

By standard arguments, the correlation functions can be calculated from the knowledge of the retarded Green function. By means of (25) we have

$$
C(i, j)=\left\langle\psi(i) \psi^{\dagger}(j)\right\rangle=\frac{a^{2}}{2(2 \pi)^{2}} \sum_{n=1}^{3} \int \mathrm{d}^{2} k e^{i \boldsymbol{k} \cdot\left(\boldsymbol{R}_{i}-\boldsymbol{R}_{j}\right)-i E_{n}(\boldsymbol{k})\left(t_{i}-t_{j}\right)} \sigma^{n}(\boldsymbol{k})\left[1+T_{n}(\boldsymbol{k})\right],
$$

where we put

$$
T_{n}(\boldsymbol{k})=\tanh \left(\frac{E_{n}(\boldsymbol{k})}{2 k_{\mathrm{B}} T}\right)
$$

We see that the calculation of the Green function requires the knowledge of the normalization matrix

$$
I(\boldsymbol{k})=\mathcal{F}\left\langle\left\{\psi(i), \psi^{\dagger}(j)\right\}\right\rangle_{\text {E.T. }}
$$

and of the $m$-matrix

$$
m(\boldsymbol{k})=\mathcal{F}\left\langle\left\{J(i), \psi^{\dagger}(j)\right\}\right\rangle_{\text {E.T. }},
$$

where $\mathcal{F}$ indicates the Fourier transform.

These quantities are calculated in the appendix and depend on a series of parameters that can be listed as follows:

1. External parameters, such as temperature $T$ and electron density $n=\left\langle c^{\dagger}(i) c(i)\right\rangle$ 
2. Model parameters $U$ and $t$;

3. Self-consistent parameters that can be calculated in terms of elements of the Green function, $\mu$ and $\Delta$;

4. Self-consistent parameters expressed as expectation values of composite fields out of the basis (17), such as $p, I_{33}^{0}, I_{33}^{\alpha}, m_{33}^{0}, m_{33}^{\alpha}$.

For the latter, the procedure of self-consistency must be fixed. In the Composite Operator Method (COM) we take advantage of this freedom and fix the parameters in such a way that the Hilbert space has the right properties to conserve the relations among matrix elements imposed by symmetry laws. In a physics dominated by a high correlation among the electrons, the main attention should be paid to the requirement that the approximation does not violate the symmetry required by the Pauli principle. Let us consider the correlation matrix (31); when we take equal points, the algebra leads to the following relations

$$
\begin{aligned}
\left\langle\xi(i) \eta^{\dagger}(i)\right\rangle & =0, \\
\left\langle\xi(i) \pi^{\dagger}(i)\right\rangle & =\left\langle c^{\alpha}(i) \xi^{\dagger}(i)\right\rangle, \\
\left\langle\eta(i) \pi^{\dagger}(i)\right\rangle & =-\left\langle c^{\alpha}(i) c^{\dagger}(i)\right\rangle
\end{aligned}
$$

among the matrix elements of the Green function. These relations constitute a set of coupled self-consistent equations which will be satisfied by an appropriate choice of the parameters.

The recovery of the Pauli principle does not exhaust all the degrees of freedom and we have place to accommodate other symmetries. An intrinsic symmetry of the Hubbard model is the pseudospin $S U(2)$ symmetry, which is nothing but invariance under the particle-hole transformation. The generators of this transformation are given by the total pseudospin operators:

$$
\begin{aligned}
P^{+} & =\sum_{i}(-)^{i} c_{\uparrow}^{\dagger}(i) c_{\downarrow}^{\dagger}(i), \\
P^{-} & =\sum_{i}(-)^{i} c_{\downarrow}(i) c_{\uparrow}(i), \\
P_{z} & =\frac{1}{2} \sum_{i}[n(i)-1] .
\end{aligned}
$$

These operators satisfy the $S U(2)$ algebra

$$
\left[P^{+}, P^{-}\right]=2 P_{z}, \quad\left[P^{ \pm}, P_{z}\right]=\mp P^{ \pm}
$$

and the Heisenberg equations

$$
\begin{aligned}
i \frac{\partial}{\partial t} P^{ \pm} & = \pm(2 \mu-U) P^{ \pm} \\
i \frac{\partial}{\partial t} P_{z} & =0
\end{aligned}
$$


Let us consider the thermal retarded Green function

$$
P^{+-}\left(t-t^{\prime}\right)=\left\langle R\left[P^{+}(t) P^{-}\left(t^{\prime}\right)\right]\right\rangle=\frac{i}{2 \pi} \int_{-\infty}^{+\infty} \mathrm{d} \omega e^{-i \omega\left(t-t^{\prime}\right)} P^{+-}(\omega) .
$$

By means of the equation of motion (38) we obtain for the correlation function

$$
\frac{1}{N}\left\langle P^{+}(t) P^{-}\left(t^{\prime}\right)\right\rangle=\frac{(n-1) e^{-i(2 \mu-U)\left(t-t^{\prime}\right)}}{1-e^{-\beta(2 \mu-U)}} .
$$

This is an exact result which relates the pseudospin correlation function to the particle number $n$ and is a manifestation of the intrinsic symmetry.

Another important symmetry is given by the conservation of the current density. By defining the charge $\rho(i)$ and current $\boldsymbol{j}(i)$ densities as

$$
\begin{aligned}
& \rho(i)=e c^{\dagger}(i) c(i), \\
& \boldsymbol{j}(i)=-i t e a^{2} c^{\dagger}(i)[\vec{\nabla}-\overleftarrow{\nabla}] c(i)
\end{aligned}
$$

we obtain, by means of the Heisenberg equation (14), the conservation law

$$
\nabla \cdot \boldsymbol{j}(i)+\frac{\partial}{\partial t} \rho(i)=0
$$

The symmetry content of the algebraic equation (43) manifests itself on the level of observation as relations among matrix elements, once a choice of the physical space of states has been made. Indeed, by defining the causal charge and current functions as

$$
\chi_{a b}(i, j)=\left\langle T\left[g_{a}(i) g_{b}(i)\right]\right\rangle=\frac{i a^{2}}{(2 \pi)^{3}} \int \mathrm{d}^{2} k \mathrm{~d} \omega e^{i \boldsymbol{k} \cdot\left(\boldsymbol{R}_{i}-\boldsymbol{R}_{j}\right)-i \omega\left(t_{i}-t_{j}\right)} \chi_{a b}(\boldsymbol{k}, \omega),
$$

where

$$
g_{a}(i)= \begin{cases}\rho(i) & \text { for } a=0 \\ j_{x}(i) & \text { for } a=x \\ j_{y}(i) & \text { for } a=y\end{cases}
$$

we can derive a series of Ward-Takahashi identities connecting current-current, charge-current and charge-charge propagators. One of those reads as follows:

$$
i a \omega \chi_{00}(\boldsymbol{k}, \omega)=\left[1-e^{-i k_{x} a}\right] \chi_{x 0}(\boldsymbol{k}, \omega)+\left[1-e^{-i k_{y} a}\right] \chi_{y 0}(\boldsymbol{k}, \omega) .
$$

In the static approximation of the Composite Operator Method the charge, current, spin, pseudospin correlation function can be connected to convolutions of single-particle propagators. This occurrence is related to a linearized dynamics together with the choice of occupation dependent electronic excitations as the basic fields [3]. Once these calculations have been performed, equations (36), (40) and (46) constitute a set of five coupled self-consistent equations which can be satisfied by an appropriate choice of the five parameters $p, I_{33}^{0}, I_{33}^{\alpha}, m_{33}^{0}, m_{33}^{\alpha}$.

Thus, we have a scheme of calculations in which it is possible to recover, through a self-consistent calculation, a series of fundamental symmetries by choosing a suitable Hilbert space.

Detailed calculations will be presented elsewhere. 


\section{Appendices}

\section{A. The normalization matrix}

From definition (33) and by means of the canonical algebra for $c$-electrons it is straightforward to see that for the paramagnetic ground state the normalization matrix has the following expression:

$$
I(\boldsymbol{k})=\left(\begin{array}{ccc}
I_{11}(\boldsymbol{k}) & 0 & I_{13}(\boldsymbol{k}) \\
0 & I_{22}(\boldsymbol{k}) & I_{23}(\boldsymbol{k}) \\
I_{13}(\boldsymbol{k}) & I_{23}(\boldsymbol{k}) & I_{33}(\boldsymbol{k})
\end{array}\right)
$$

with

$$
\begin{aligned}
& I_{11}(\boldsymbol{k})=1-\frac{n}{2}, \\
& I_{13}(\boldsymbol{k})=\Delta+\left(p-I_{22}\right) \alpha(\boldsymbol{k}), \\
& I_{22}(\boldsymbol{k})=\frac{n}{2} \\
& I_{23}(\boldsymbol{k})=-\Delta-p \alpha(\boldsymbol{k}), \\
& I_{33}(\boldsymbol{k})=I_{33}^{0}+\alpha(\boldsymbol{k}) I_{33}^{\alpha} .
\end{aligned}
$$

The quantities introduced in (A.2) are defined:

$$
\begin{aligned}
n & =\left\langle c^{\dagger}(i) c(i)\right\rangle=2\left[1-\left\langle\xi(i) \xi^{\dagger}(i)\right\rangle-\left\langle\eta(i) \eta^{\dagger}(i)\right\rangle\right], \\
\Delta & =\left\langle\xi^{\alpha}(i) \xi^{\dagger}(i)\right\rangle-\left\langle\eta^{\alpha}(i) \eta^{\dagger}(i)\right\rangle, \\
p & =\frac{1}{4}\left\langle n_{\mu}^{\alpha}(i) n_{\mu}(i)\right\rangle-\left\langle\left[\xi_{\uparrow}(i) \eta_{\downarrow}(i)\right]^{\alpha} \eta_{\downarrow}^{\dagger}(i) \xi_{\uparrow}^{\dagger}(i)\right\rangle,
\end{aligned}
$$

$n$ is the average number of electrons per site; $\Delta$ and $p$ are static correlation functions between nearest neighbour sites. In particular, parameter $p$ describes the inter-site charge, spin and pair correlations. In the calculation of $I_{33}(\boldsymbol{k})$ only the nearest neighbour contributions are retained

$$
\left\langle\left\{\pi(i), \pi^{\dagger}(j)\right\}\right\rangle \cong \delta_{i j} I_{33}^{0}+\alpha_{i j} I_{33}^{\alpha} .
$$

\section{B. The $m$-matrix}

First of all we note that time translational invariance requires the $m$-matrix to be hermitian

$$
m(\boldsymbol{k})=\left(\begin{array}{ccc}
m_{11}(\boldsymbol{k}) & m_{12}(\boldsymbol{k}) & m_{13}(\boldsymbol{k}) \\
m_{12}(\boldsymbol{k}) & m_{22}(\boldsymbol{k}) & m_{23}(\boldsymbol{k}) \\
m_{13}(\boldsymbol{k}) & m_{23}(\boldsymbol{k}) & m_{33}(\boldsymbol{k})
\end{array}\right) .
$$

From definition (34) and by making use of expressions (20-21) for the source current it is possible to calculate

$$
m_{11}(\boldsymbol{k})=-[\mu+4 t \alpha(\boldsymbol{k})] I_{11}-4 t I_{13}
$$




$$
\begin{aligned}
& m_{12}(\boldsymbol{k})=-4 t \alpha(\boldsymbol{k}) I_{22}-4 t I_{23}, \\
& m_{13}(\boldsymbol{k})=-[\mu+4 t \alpha(\boldsymbol{k})] I_{13}-4 t\left[\alpha(\boldsymbol{k}) I_{23}+I_{33}\right], \\
& m_{22}(\boldsymbol{k})=-(\mu-U) I_{22}+4 t I_{23}, \\
& m_{23}(\boldsymbol{k})=-(\mu-U) I_{23}+4 t I_{33}, \\
& m_{23}(\boldsymbol{k})=m_{33}^{0}+\alpha(\boldsymbol{k}) m_{33}^{\alpha} .
\end{aligned}
$$

In the calculation of $m_{33}(\boldsymbol{k})$ only the nearest neighbour contributions are retained

$$
\left\langle\left\{J_{3}(i), \pi^{\dagger}(j)\right\}\right\rangle \cong \delta_{i j} m_{33}^{0}+\alpha_{i j} m_{33}^{\alpha} .
$$

\section{References}

1. E.Dagotto. Correlated electrons in high-temperature superconductors. Rev. Mod. Phys. 66, 763 (1994); Z.-X.Shen, D.S.Dessau. Electronic structure and photoemission studies of late transition-metal oxides - Mott insulators and high-temperature superconductors. Phys. Rep. 253, 1 (1995); R.S.Markiewicz. A survey of the van hove scenario for high$T_{c}$ superconductivity with special emphasis on pseudogaps and striped phases, condmat/9611238.

2. N.Plakida. High-temperature superconductivity. (Springer-Verlag, 1995); Models and phenomenology for conventional and high- $T_{c}$ superconductivity, Proceedings of the Varenna "E.Fermi" school edited by G.Iadonisi, J.R.Schrieffer and M.Chiofalo (1997).

3. F.Mancini, S.Marra, H.Matsumoto. Doping dependence of on-site quantities in the twodimensional Hubbard model. Physica C 244, 49 (1995); Energy and chemical potential in the two-dimensional Hubbard model. Ibid. 250, 184 (1995); F.Mancini, D.Villani, H.Matsumoto. Incommensurate magnetism in cuprate materials. Phys. Rev. B 57, 6145 (1998).

4. F.Mancini. Conservation of spectral moments in the $n$-pole approximation, condmat/9803276.

5. O.K.Kalashnikov, E.S.Fradkin. The method of spectral densities in quantum statistical mechanics. Sov. Phys. JETP 28, 317 (1969); G.Geipel, W.Nolting. Ferromagnetism in the strongly correlated Hubbard model. Phys. Rev. B 38, 2608 (1988); W.Nolting, W.Borgel. Band magnetism in the Hubbard model. Phys. Rev. B 39, 6962 (1989).

6. A.Avella, F.Mancini, D.Villani, L.Siurakshina, V.Yu.Yushankhai. The Hubbard model in the two-pole approximation. Int. J. Mod. Phys. B 12, 81 (1998).

7. D.J.Rowe. Equations-of-motion method and the extended shell model. Rev. Mod. Phys. 40, 153 (1968).

8. J.Hubbard. Electron correlations in narrow energy bands. Proc. Roy. Soc. A 276, 238 (1963). 


\title{
Симетрії у фізиці сильно скорельованих електронних систем
}

\author{
Ф.Манчіні, А.Авелла
}

Університет Салерно, Факультет фізичних наук ім. Е.Р. Каіаніелло, 84081 Бароніссі, Салерно, Італія

Отримано 11 червня 1998 р.

Системи з сильною електронною кореляцією потребують розвитку нових теоретичних схем з метою опису їх незвичайних і неочікуваних властивостей. Звичайні схеми теорії збурень $є$ неадекватні і $є$ потреба у введенні нових концепцій. У нашому підході - Методі Композитного Оператора - є можливим відтворити в рамках самоузгодженого розрахунку ряд фундаментальних симетрій шляхом вибору відповідного гільбертового простору.

Ключові слова: системи з сильною електронною кореляцією, модель Хаббарда, симетрії, метод композитного оператора

PACS: $71.10 .-w, 71.10 . F d, 71.27 .+a$ 